\title{
2.7 VECTORS
}

\author{
Thomas Burkot and Moses Bockarie**
}

\section{Summary of Prioritized Research Needs}

1) Refine and evaluate the new tools for determining LF infection rates in mosquitoes,

2) Develop and evaluate new tools for estimating the rate of contact between human and mosquito populations,

3) Evaluate vector control strategies based on knowledge of the vectors' biology for impact on LF transmission, including

a) insecticide impregnated mosquito nets on LF transmission by Anopheles and Culex vectors,

b) breeding site reduction strategies on LF transmission by Aedes vectors,

c) polystrene beads and Bacillus sphaericus on LF transmission by Culex vectors,

4) Conduct operational research on how to integrate successful vector control strategies for LF into ongoing programs for other vector borne diseases (malaria, dengue, etc.).

\subsubsection{Overview: Vector Control for Filariasis}

Until the discovery of the anti-filarial properties of DEC, filariasis control programs were based on suppressing transmission though vector control. This approach took advantage of the relatively inefficient nature of filariasis transmission and the absence of significant reservoir hosts. ${ }^{1}$ The real challenge was in identifying control measures that targeted weaknesses in the biology of the vectors. When appropriate vector control was applied, success in controlling and even eliminating filariasis was achieved. For example, environmental sanitation reduced the breeding sites of the Culex vectors of filariasis in Australia ${ }^{2}$ and Japan, $^{3}$ resulting in the eventual elimination of LF from these two countries. Further proof of the success of vector control was seen when residual wall straying with DDT for malaria control inadvertantly eliminated LF from the Solomon Islands ${ }^{4,5}$ and parts of Papua New Guinea. ${ }^{6}$

The GPELF is based on annual MDA with albendazole and either DEC or ivermectin. ${ }^{7}$ Suppression of transmission though vector control should, however, be considered an important adjunct or supplement to MDA programs. Studies have already shown that MDA programs integrated with vector control require fewer annual rounds of MDA to achieve the same level of success. ${ }^{8}$ If the effectiveness of MDAs decreases because of poor population compliance or the development of resistance by the parasite to DEC, ivermectin or albendazole, the importance of vector control as a supplemental measure to suppress transmission and thereby ensure LF elimination would be even greater. ${ }^{9}$

The challenge for applying vector control to filariasis elimination programs lies principally in knowing where and when it should be used for maximal cost-effectiveness. Implemen-

\footnotetext{
** Other contributors in this working group are listed in Annex 2.
}

tation of vector control is complicated by the large number of vector species found in endemic countries. ${ }^{10}$ Cost-effective vector control programs must be based on 1) detailed understanding of the ecology of the major vectors in each area, as well as 2) methods for monitoring the cost and impact of vector control. Furthermore, the effective control strategies identified must be integrated with existing vector-borne disease control programs.

\subsubsection{Research Needs}

Indicators to evaluate transmission interruption by vector monitoring (including xenomonitoring).

Evaluating the suppression of LF transmission can be undertaken in either/both the human and mosquito populations. Because of the long persistence of the parasite and antigenemia (measured by ICT) in humans, there is particular value in assessing infection rates in the mosquito population as an almost real time indicator of ongoing transmission (especially early in MDA programs) or the absence of transmission (hopefully at the conclusion of such MDA activities).

Xenomonitoring (particularly using PCR to detect parasite DNA) is especially effective in following the rate at which people infect mosquitoes with $\mathrm{mf}$, while defining the level of transmission from mosquito to humans requires detecting infective stage larvae (L3) in the mosquito. ${ }^{11}$ Both give real time estimates of transmission and both require sensitive methods for detecting $\mathrm{mf}$ and L3s in mosquitoes. Also, very importantly, both approaches require careful, effective sampling strategies to define the infection levels in the mosquito populations well enough to estimate both human attack rates and LF infection rates. Important ethical concerns about the use of (potentially infectious) mosquito landing catches as measures of biting rates on humans have also been raised. There are, therefore, important areas of research to be addressed

- to develop new, standardized methods for sampling mosquito populations that will yield samples representative of the entire population and be widely applicable,

- to compare PCR and dissection-based assays to determine the basis for potentially discordant results,

- to develop new techniques for determining infection rates in mosquitoes through large-scale sampling,

- to validate new techniques to determine infection rates in mosquitoes through multicountry trials (i.e., in multiepidemiologic settings),

- to develop new methods for estimating the size of biting mosquito populations.

Tailoring vector control strategies to the specific needs of national MDA-based LF elimination programs.

Additional methods for suppressing transmission of LF are needed as adjuncts or supplements to the MDA-based pro- 
grams. ${ }^{12}$ Low MDA coverage of endemic populations or even possible development of resistance by the parasite to DEC, albendazole, or ivermectin could threaten the success of national LF elimination programs. In addition, the danger of applying MDA programs in loiasis-endemic areas necessitates the development of alternative strategies that can reduce microfilarial densities to levels where significant adverse reactions following MDA are not seen. ${ }^{13}$

The historic success of vector control programs on LF transmission argues for the evaluation of new mosquito control strategies for augmenting LF programs. Since LF is transmitted by a wide range of mosquito genera in different geographic areas, multiple control strategies will need to be developed that are tailored to different epidemiologic conditions. While ITNs are a mainstay of the Roll Back Malaria program, ${ }^{14,15}$ their potential impact on filariasis transmission by both Anopheles and Culex vectors requires further evaluation. ${ }^{15,16}$ The use of expanded polystyrene beads in water filled pits to act as a physical barrier to both ovipositing Culex mosquitoes and emerging adults has been shown to drastically reduce biting populations of Culex LF vectors. ${ }^{17}$ When the use of polystyrene beads for Culex vector control was implemented with MDA, resurgence of LF after MDA ended was prevented. The potential of biological, chemical, and source reduction strategies to interrupt LF transmission by Aedes vectors has been demonstrated in limited areas, but the suitability of these approaches to other areas must be explored and the operational barriers to widespread implementation identified and overcome. ${ }^{18}$ The potential of these and other mosquito control methods to act as adjunct transmission suppression measures to MDA to ensure LF elimination needs to be substantiated. Therefore, it is necessary that

- maps of the distribution of LF vectors be developed for regions needing adjunctive vector control,

- information on the biology of the important LF vectors be determined (breeding sites, flight range, host blood feeding preferences, resting and biting activities) where it is unknown and relevant to the control strategy to be implemented,

- the efficacy of ITNs be evaluated for night- biting Culex vectors,

- the reasons for use and lack of use of ITNs by LF-infected people be understood (are people who do not participate in MDA also more likely to not use an ITN?),

- the impact of community-based breeding-source-reduction campaigns on LF transmission be evaluated in areas where Aedes are the primary vectors,

- appropriate strategies for interrupting LF transmission by Culex be developed and evaluated (including use of ITNs, polystyrene beads in pit latrines, etc.),

- strategies for interrupting LF transmission by Aedes be evaluated (including source reduction, use of insecticide impregnated materials, etc.),

- measurement of the impact of vector control on the number of MDAs required to interrupt LF transmission be determined,

- the possibility be assessed that vector control alone or along with targeted chemotherapy can eliminate LF transmission when only residual pockets of infection remain after five or more annual MDAs.
Integrating LF vector control activities into existing national vector control programs.

Sustained suppression of LF transmission will depend on integrating LF activities, including those for vector control, with other local public health programs. For the vector control components of LF programs, integration with malaria and dengue control programs would be logical. ${ }^{14,15,18}$ Operational research to understand how to motivate communities to undertake vector control and strategies for health communication will be essential for successful implementation of community-based anti-mosquito interventions. To accomplish this integration, however, it is necessary

- to identify the implementable approaches to vector control that are common for LF and other vector-borne infections occurring in LF-endemic areas,

- to evaluate the impact of current vector control measures for malaria, dengue, etc. on the transmission of LF,

- to undertake KAP surveys to assess the understanding of the target population on LF transmission and the acceptability of mosquito control strategies, and

- to develop health communications strategies based on the KAP survey results with pretesting and evaluation after dissemination.

\subsubsection{References}

1. Ottesen EA, Duke BO, Karam M, Behbehani K, 1997. Strategies and tools for the control/elimination of lymphatic filariasis. Bull World Health Organ 75: 491-503.

2. Boreham PFL, Marks EN, 1986. Human filariasis in Australia: introduction, investigation and elimination. Proc $R$ Soc Queensland 97: 23-52.

3. Sasa M, 1976. Human Filariasis: A Global Survey of Epidemiology and Control. Tokyo: University Park Press, 458-508,

4. Webber RH, 1977. The natural decline of Wuchereria bancrofti infection in a vector control situation in the Solomon Islands. Trans R Soc Trop Med Hyg 71: 396-400.

5. Webber RH, 1979. Eradication of Wuchereria bancrofti infection through vector control. Trans $R$ Soc Trop Med Hyg 73: 722-724.

6. Bockarie M, 1994. Can lymphatic filariasis be eradiated in Papua New Guinea? P N G Med J 37: 61-64.

7. Molyneux DH, Zagaria N, 2002. Lymphatic filariasis elimination: progress in global programme development. Ann Trop Med Parasitol 96 (Suppl 2): S15-S40.

8. Sunish IP, Rajendran R, Mani TR, Munirathinam A, Tewari SC, Hiriyan J, Gajanana A, Satyarayana K, 2002. Resurgence in filarial transmission after withdrawal of mass drug administration and the relationship between antigenaemia and microfilaraemia-a longitudinal study. Trop Med Int Health 7: 59-69.

9. Burkot TR, Ichimori K, 2002. The Pacific program for the elimination of lymphatic filariasis: will mass drug administration be enough? Trends Parasitol 18: 109-115.

10. Raghavan HGS, 1961. The vectors of human infections by Wuchereria species in endemic areas and their biology. Bull World Health Organ 24: 177-195.

11. Williams SA, Laney SJ, Bierwert LA, Saunders LJ, Boakye DA, Fischer P, Goodman D, Helmy H, Hott SL, Vasuki V, Lammie PJ, Pichart C, Ramzy RMR, Ottesen EA, 2002. Development and standardization of a rapid, PCR-based method for the detection of Wuchereria bancrofti in mosquitoes, for xenomonitoring the human prevalence of Bancroftian filariasis. Ann Trop Med Parasitol 96 (Suppl 2): S41-S46.

12. Burkot TR, Taleo G, Toeaso V, Ichimori K, 2002. Progress towards and challenges for the elimination of filariasis from $\mathrm{Pa}$ cific-island communities. Ann Trop Med Parasitol 96 (Suppl 2): S61-S69. 
13. Chippauz J-P, Boussinesq M, Gardon J, Gardon-Wendel N, Ernould J-C, 1996. Severe adverse reaction risks during mass treatment with ivermectin in loiasis-endemic areas. Parasitol Today 12: 448-450.

14. Manga L, 2002. Vector-control synergies, between 'Roll Back Malaria' and the Global Programme to Eliminate Lymphatic Filariasis, in the African region. Ann Trop Med Parasitol 96 (Suppl 2): S129-S132.

15. Prasittisuk C, 2002. Vector-control synergies, between 'Roll Back Malaria' and the Global Programme to Eliminate Lymphatic Filariasis, in south-east Asia. Ann Trop Med Parasitol 96 (Suppl 2): S133-S137.
16. Pedersen EM, Mukoko DA, 2002. Impact of insecticide-treated materials on filaria transmission by the various species of vector mosquito in Africa. Ann Trop Med Parasitol 96 (Suppl 2): S91-S95.

17. Curtis CF, Malecela-Lazaro M, Reuben MR, Maxwell C, 2003. Use of floating layers of polystyrene beads to control populations of the filarial vector, Culex quinquefasciatus. Ann Trop Med Parasitol 96 (Suppl 2): S97-S104.

18. Lardeux F, Riviere F, Sechan Y, Loncke S, 2002. Control of the Aedes vectors of the dengue viruses and Wuchereria bancrofti: the French Polynesian experience. Ann Trop Med Parasitol 96 (Suppl 2): S105-S116. 